\title{
Metasurface Design Based on S-Shaped Split Ring Resonator for Linear-to-Circular Polarization Conversion
}

\author{
Erfeng Li \\ Dept. of Electrical \& Electronic Eng. \\ Auckland University of Technology \\ Auckland, New Zealand \\ brandt.li@aut.ac.nz
}

\author{
Xue Jun Li \\ Dept. of Electrical \& Electronic Eng. \\ Auckland University of Technology \\ Auckland, New Zealand \\ xuejun.li@aut.ac.nz
}

\author{
Boon-Chong Seet \\ Dept. of Electrical \& Electronic Eng. \\ Auckland University of Technology \\ Auckland, New Zealand \\ boon-chong.seet@aut.ac.nz
}

\begin{abstract}
In this paper, we proposed a single layered metasurface linear-to-circular polarization converter based split ring resonator structure. The periodic unit cell of the converter includes nested "S-shaped" and "C-shaped" rings. Simulated results show that the converter can reflect incident waves with equal magnitude and $90^{\circ}$ phase difference to achieve circular polarization conversion and at $12.4 \mathrm{GHz}$, linear polarized wave will be converted to left-handed circular polarized wave; the conversion efficiency is above $90 \%$; the converted waves has an axial ratio below $3 \mathrm{~dB}$.
\end{abstract}

Index Terms-Split ring resonator, metasurface, linear to circular polarization

\section{INTRODUCTION}

For an antenna, polarization is one of the most important characteristics because its performance can vary significantly due to the inherent polarization-sensitive property in the materials involved in electromagnetic wave propagation. Therefore, polarization conversion of electromagnetic waves becomes significant for certain practical applications, especially linear-tocircular (LTC) polarization conversion [1], [2]. Conventional polarization manipulation methods are normally achieved via the application of complex structures, such as birefringent crystalline liquid or crystals. These converters normally come in large size and thickness, making it difficult for compact system integration. Additionally, low efficiency is another drawback for the conventional polarization converters [1].

In recent years, metamaterial has become popular, providing new ways for polarization conversion. Metamaterials can be defined as artificially crafted composite materials whose properties are derived from internal microstructure, rather than chemical composition found in natural materials. For example, it typically requires multiple stacks of material layers to realise the fascinating functionalities of metamaterials. This not only brings a lot of challenges in nanofabrication, but also leads to extensive losses. Many metamaterials consist of complex metallic wires and other structures that require sophisticated fabrication technology and are difficult to assemble [3], [4]. To overcome those obstacles faced by metamaterials, metasurfaces are proposed and they feature thin films composed of individual elements. Metasurface generally refers to a periodic or quasi-periodic planar array of sub-wavelength units [4]. By applying different types of unit cell structure and manipulating its parameters, the metasurface will demonstrate different characteristics in reflecting electromagnetic waves, in terms of frequencies, amplitudes, phases and bandwidths. The thickness of metasurface is also relatively smaller depending on the dielectric material selected [1], [2].

In [5], Zou et al. proposed an LTC converter based on substrate integrated waveguide cavity unit cell for $15 \mathrm{GHz}$ $15.7 \mathrm{GHz}$. However, the feasibility of fabrication is high due to the structure of metallic via holes, which can also increase the fragility of the entire surface, given the number and density of the via holes. In [6], a dual-band circular polarization converter based on anisotropic metamaterial was proposed for $4.5 \mathrm{GHz}$ and $7.9 \mathrm{GHz}$ application, however it employed a three-layer structure, which increased the difficulty for fabrication.

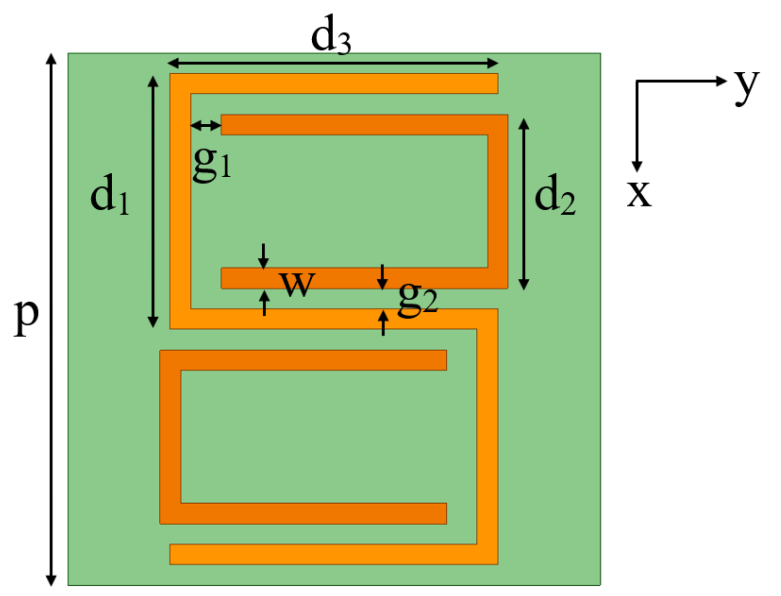

Fig. 1. Antenna layout and dimensions $\left(p=13 \mathrm{~mm}, d_{1}=5.75 \mathrm{~mm}, d_{2}=\right.$ $\left.4.25 \mathrm{~mm}, d_{3}=8 \mathrm{~mm}, w=0.5 \mathrm{~mm}, g_{1}=0.75 \mathrm{~mm}, g_{2}=0.5 \mathrm{~mm}\right)$

In this paper, a single layered metasurface based LTC polarization converter is proposed for application at $12.4 \mathrm{GHz}$. The unit cell of the design is based on " $\mathrm{S}$ "-shaped and " $\mathrm{C}$ "- 




Fig. 2. Boundary conditions and Floquet port setup in simulation

shaped split ring resonator (SRR) structure. It demonstrates high conversion efficiency and the converted circular polarized wave has axial ratio (AR) below $3 \mathrm{~dB}$.

\section{Unit Cell Design}

The proposed LTC polarization converter design has a periodic unit cell with "S"-shaped SSR, which operates at 12.5 GHz. It is designed on a substrate of Rogers RT/droid 5880 of thickness $t=1.575 \mathrm{~mm}$ and dielectric constant $\varepsilon_{r}=2.2$. The radiating layer is an anisotropic "S"-shaped SSR. There are two centrosymmetrical " $\mathrm{C}$ "-shaped inner rings inside the opening of the "S". The unit cell has a periodicity of $p=13$ $\mathrm{mm}$ and its dimensional parameters are illustrated in Fig. 1.

Theoretically, when an incident wave reaches on the surface of polarization converter, it will be reflected with a certain magnitude and phase. For instance, given a downward $y$ polarized incident wave, the reflected wave can be expressed by equation (1) [1]:

$$
\begin{aligned}
\boldsymbol{E}_{r} & =E_{x r} \boldsymbol{e}_{x}+E_{y r} \boldsymbol{e}_{y} \\
& =E_{x} \exp \left(j \phi_{x y}\right) \boldsymbol{e}_{x}+E_{y} \exp \left(j \phi_{y y}\right) \boldsymbol{e}_{y}
\end{aligned}
$$

In equation (1), $r_{x y}=\left|E_{x r} / E_{y i}\right|$ and $r_{y y}=\left|E_{y r} / E_{y i}\right|$ represent the reflection coefficient magnitudes of $y$-to- $x$ and $y$-to- $y$ polarization conversion, respectively, whereas $\phi_{x y}$ and $\phi_{y y}$ are the corresponding phases. To achieve an LTC polarization conversion, two conditions have to be fulfilled, i.e., $r_{x y}=r_{y y}$ and $\Delta \phi=\phi_{y y}-\phi_{x y}=2 n \pi \pm \pi / 2$, where $\Delta \phi$ is the phase difference between $E_{x r}$ and $E_{y r}$. When $\Delta \phi$ has a " $-\pi / 2$ " component, it indicates the right-hand circular polarization (RHCP), whereas a " $+\pi / 2$ " component indicates left-hand circular polarization (LHCP).

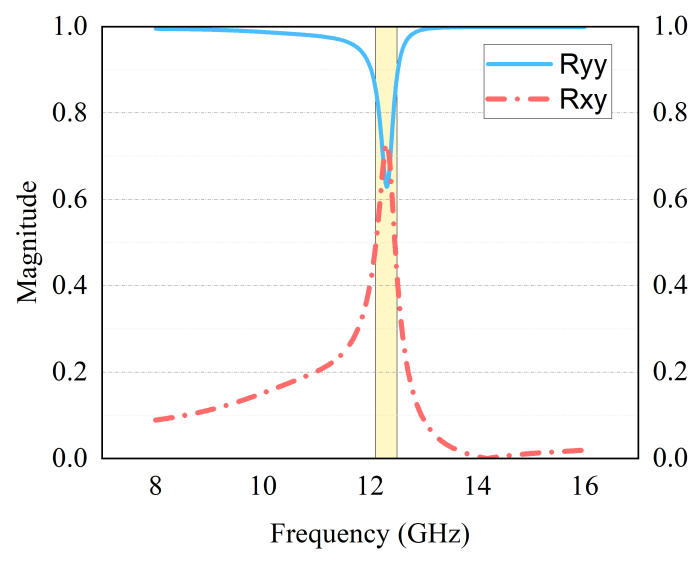

Fig. 3. Magnitudes of reflection coefficients

\section{Simulation AND Results}

The design was simulated using High Frequency Structure Simulator (HFSS). In the simulation, master and slave boundary conditions were set up for infinite array approximation and Floquet port was assigned to the top face of the radiation box, as shown in Fig. 2. At $12.4 \mathrm{GHz}$, the magnitudes of reflection coefficient are approximately equal (value of 0.70 ) with a phase difference of $-270^{\circ}$ (or $90^{\circ}$ ), which are shown in Fig. 3 and Fig. 4, respectively. Hence, the reflected wave is an LHCP wave.

$$
\begin{gathered}
I=\left|r_{x y}\right|^{2}+\left|r_{y y}\right|^{2} \\
Q=\left|r_{y y}\right|^{2}-\left|r_{x y}\right|^{2} \\
U=2\left|r_{x y}\right|\left|r_{y y}\right| \cos \Delta \phi \\
V=2\left|r_{x y}\right|\left|r_{y y}\right| \sin \Delta \phi
\end{gathered}
$$

To further evaluate the performance of the proposed LTC polarization converter, four Stroke parameters are introduced, shown in equation (2) to (5) [1]. To describe the degree to which the polarization has been converted, ellipticity is defined as $e=V / I$. When $e=1$, it is a perfect LHCP conversion; while $e=-1$, it indicates a perfect RHCP conversion. The ellipticity is close to 1 at frequency of $12.4 \mathrm{GHz}$, as shown in Fig. 4, which means the reflected wave is close to be perfectly LHCP wave.

Another two angles $\alpha$ and $\beta$ are defined by the Stroke parameters as expressed in equation (6) and (7), where $\alpha$ is the polarization azimuth angle while $\beta$ is the ellipticity angle. Additionally, from the given phase difference $\Delta \phi$, the axial ratio (AR) could be calculated by equation (5) [7] and it can be seen from Fig. 5 that the AR of the proposed design was below $3 \mathrm{~dB}$, which indicates it is qualified for circular polarization. 




Fig. 4. Ellipticity and phase difference



Fig. 5. Axial ratio and efficiency

$$
\tan 2 \alpha=U / Q
$$

$$
\sin 2 \beta=V / I
$$

$$
\mathrm{AR}=\left(\frac{\left|r_{y y}\right|^{2}+\left|r_{x y}\right|^{2}+\sqrt{a}}{\left|r_{y y}\right|^{2}+\left|r_{x y}\right|^{2}-\sqrt{a}}\right)^{1 / 2}
$$$$
a=\left|r_{y y}\right|^{4}+\left|r_{x y}\right|^{4}+2\left|r_{y y}\right|^{2}\left|r_{x y}\right|^{2} \cos (2 \Delta \phi)
$$

$$
\eta=\left(\left|E_{x r}\right|^{2}+\left|E_{y r}\right|^{2}\right) /\left|E_{y i}\right|^{2}=\left|r_{x y}\right|^{2}+\left|r_{y y}\right|^{2}
$$

Another parameter, energy conversion efficiency $\eta$, is given by equation (10), and the efficiency of proposed design is shown in Fig. 5. It can be noticed that the efficiency reached above $90 \%$ at $12.4 \mathrm{GHz}$.

\section{Conclusion}

In this paper, a novel single-layered LTC polarization converter was proposed, and designed on metasurface based on a periodic SRR. Simulated results show high conversion rate and efficiency. The AR of converted waves is also below 3 $\mathrm{dB}$. The future work includes fabrication of the design and realising multi-band features.

\section{REFERENCES}

[1] Y. Li, J. Zhang, S. Qu, J. Wang, L. Zheng, Y. Pang, Z. Xu, and A. Zhang, "Achieving wide-band linear-to-circular polarization conversion using ultra-thin bi-layered metasurfaces," Journal of Applied Physics, vol. 117, no. 4, p. 044501, Jan. 2015. [Online]. Available: http://aip.scitation.org/doi/10.1063/1.4906220

[2] Y. Jiang, L. Wang, J. Wang, C. N. Akwuruoha, and W. Cao, "Ultra-wideband high-efficiency reflective linear-to-circular polarization converter based on metasurface at terahertz frequencies," Optics Express, vol. 25, no. 22, p. 27616, Oct. 2017. [Online]. Available: https://www.osapublishing.org/abstract.cfm?URI=oe-25-22-27616

[3] Y. Luo, K. Qin, H. Ke, B. Xu, S. Xu, and G. Yang, "Active metamaterial antenna with beam scanning manipulation based on a digitally modulated array factor method," IEEE Transactions on Antennas and Propagation, vol. 69, no. 2, pp. 1198-1203, 2021.

[4] J. Wang, Y. Li, Z. H. Jiang, T. Shi, M.-C. Tang, Z. Zhou, Z. N. Chen, and C.-W. Qiu, "Metantenna: When metasurface meets antenna again," IEEE Transactions on Antennas and Propagation, vol. 68, no. 3, pp. 1332-1347, 2020.

[5] Y. Zuo, J. Shi, J. Li, Z. Jin, R. Chen, and Y. Zhang, "A linear to circular polarization converter based on substrate integrated waveguide cavities," in 2019 Cross Strait Quad-Regional Radio Science and Wireless Technology Conference (CSQRWC), 2019, pp. 1-3.

[6] Y. Cheng, J. Fan, H. Luo, and F. Chen, "Dual-band and high-efficiency circular polarization convertor based on anisotropic metamaterial," IEEE Access, vol. 8, pp. 7615-7621, 2020.

[7] B. Lin, L. Lv, J. Guo, Z. Liu, X. Ji, and J. Wu, "An ultra-wideband reflective linear-to-circular polarization converter based on anisotropic metasurface," IEEE Access, vol. 8, pp. 82 732-82 740, 2020. 